\title{
Alignment analysis of urban railways based on passenger travel demand
}

\author{
J. L. E. Andersen \& A. Landex \\ Department of Transport, Technical University of Denmark, Denmark
}

\begin{abstract}
Planning of urban railways like Metro and especially Light Rail Transit often result in multiple alignment alternatives from where it can be difficult to select the best one. Travel demand is a good foundation for evaluating a railway alignment for its ability to attract passengers. Therefore, this article presents a computerised GIS based methodology that can be used as decision support for selecting the best alignment. The methodology calculates travel potential within defined buffers surrounding the alignment. The methodology has three different approaches depending on the desired level of detail: the simple but straightforward to implement line potential approach that perform corridor analysis, the detailed catchment area analysis based on stops on the alignment and the refined service area analysis that uses search distances in street networks. All three approaches produce trustworthy results and can be applied as decision support in different stages of the urban railway alignment planning.

Keywords: public transport, urban railways, metro, light rail transit, alignment, catchment area, service area, travel demand, travel potential, GIS, planning.
\end{abstract}

\section{Introduction}

Conventional railways are usually large and rigid with few degrees of freedom in planning of alignments. This is due to the characteristics of such rail systems: high average stop distance and stop positioning dominated by strategic requirements of service (e.g. stop in the big cities the railway passes). However, smaller flexible urban railways like Metro and especially Light Rail Transit (LRT) have much lower average stop distance and the stop positioning may not be evident when consistently running in build-up areas. Therefore, it is often seen that the screening phase of a new urban railway consists of multiple 
strategic alignment options or alternatives (e.g. see [1]). It may be difficult to choose the best alignment between multiple high quality alternatives and a decision support tool is often required. Traffic modelling of each alternative will usually provide the best decision base. However, traffic modelling is very time consuming and expensive and is, therefore, usually not introduced until a later phase of the planning process where the number of alternatives are low or nonexisting. A quick-to-implement decision support for selecting alignment alternatives that can be used in an earlier planning phase is, therefore, desirable.

Among other important decision elements of the urban railway alignment planning such as transfers, travel time and construction cost travel demand has the highest influence. This is because travel demand constitutes the customer base in the surrounding areas of a railway line. Therefore, a decision support methodology based on passenger travel demand to aid the selection of the best alignment between multiple others is relevant. In the following such methodology - with different approaches depending on the level of detail - is presented and evaluated for its applied use in the planning of alignments for urban railways.

A case example will be introduced to show the applied use of the methodology. The case example is based on a light rail solution since this type of urban railway gives rise to most alignment alternatives.

\subsection{Introduction to case example}

The case example is taken from Copenhagen, Denmark and deals with a light rail proposal going from the city centre to the main airport running on the northern part of the island of Amager. The focus area of the case can be seen in figure 1.

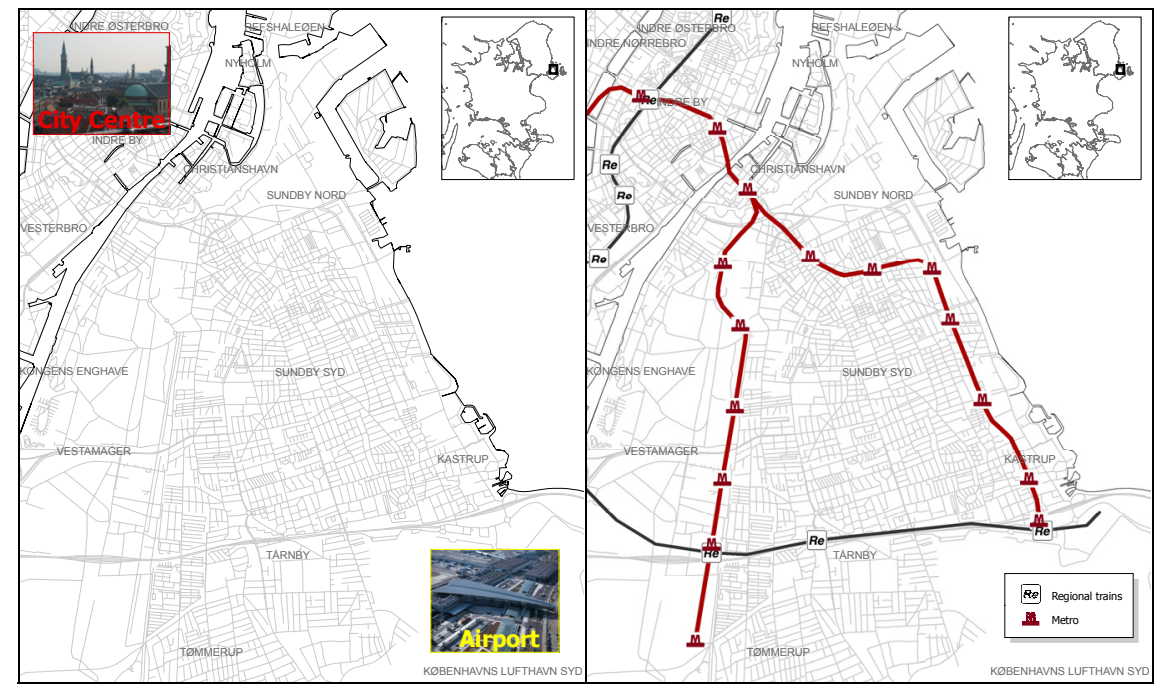

Figure 1: Focus area of case example - the northern part of the island of Amager (left side), and the existing high quality public transport in the focus area (right side). 
There are already rail connections between the city centre and the main airport by regional trains and Metro. However, these are relatively fast connections with few stops whereas a light rail solution is intended to service more locally on the island of Amager and will not (and cannot) compete for travellers going all the way between the city centre and the main airport.

\section{Passenger travel demand}

Travel demand can be used to investigate the need for public transport services in specific areas. Travel demand for public transport can be an indication of potential passengers hence the term passenger travel demand. There are many different factors that affect travel demand. Some are very dominant and have a regular impact (residences, workplaces, student places etc.) while some are only dominant in a time specific period thus having an irregular impact (stadiums, beaches, amusement parks etc.). Furthermore, the passenger travel demand is dependant on the socio-economic composition of the examined area (car ownership, income, ages, family types, driver licenses etc.). For instance, the passenger travel demand is more likely to be utilized in areas with low car ownership than in areas with high car ownership.

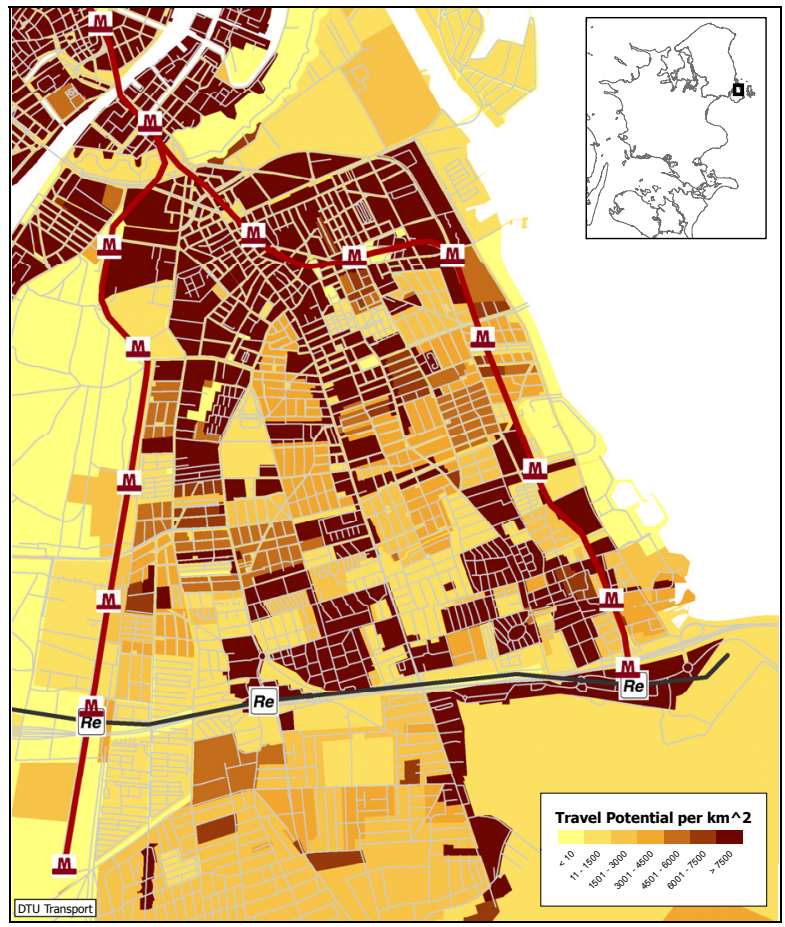

Figure 2: $\quad$ Travel potential within the focus area. 
In applied analysis of public transport it can be difficult to include all travel demand factors. Therefore, a simplified - but relatively good and understandable - delimitation such as travel potential can be used. Travel potential includes the most important and regular impact on travel demand: Population and workplaces. To get one overall expression of these two factors they can be weighted together in a mutual relation:

$$
\text { Travel potential }=\text { Population }+1.75 \times \text { Workplaces }
$$

Studies have shown that a workplace gives rise to $75 \%$ more traffic than an inhabitant mainly due to work travel [2] why the workplaces are given a higher weight in equation (1).

The travel potential for different areas can be visualized and especially travel potential density is relevant to show on maps as seen in figure 2 .

\section{Alignment alternatives}

Planning urban railways, and especially LRT, can result in multiple alignment alternatives. Usually the end stops are given but how to get from end to end can vary and is depending on various conditions. Aside from travel demand it can be

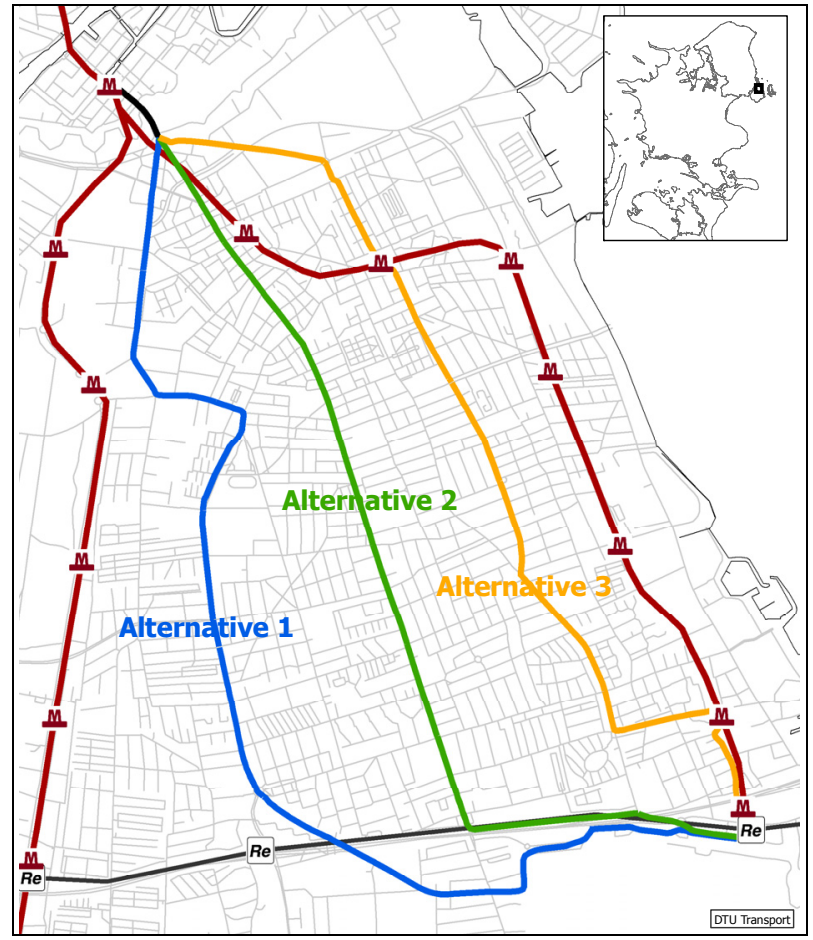

Figure 3: Light rail alignment proposals (alternatives) in the focus area. 
with regard to factors such as connection to other public transport lines (feeder lines), travel distance/time, construction cost, special location service (hospitals, stadiums etc.) and - especially for LRT - availability of space (road width etc.). Although many alignment alternatives can be opted out in an early phase there will nearly always be cases where alternatives offhand appear equally good and this are when decision support is needed to determine the final alignment.

\subsection{Case example: Alignment alternatives}

In the case example three different alignment alternatives has been chosen for investigation, cf. figure 3 . The placing of the alignment revealed more variations within each of the three alternatives just as the three alignment alternatives could be combined in various sequences. All these different variations have been deselected for this purpose since they produced too many alternatives for the case example.

\subsection{Stop positioning}

Methods to select between alignment alternatives without considering stops exist. But the most accurate analyses are performed on stops since they are the passengers' access and egress to the railway system. Therefore, it can be relevant

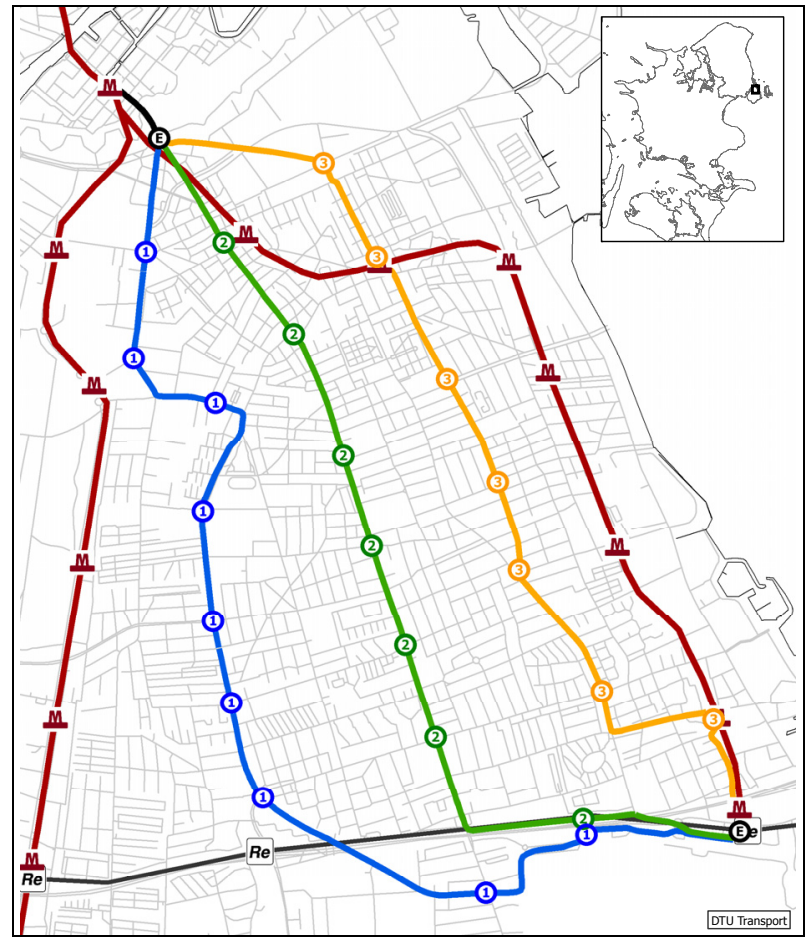

Figure 4: Stop positioning on the three alignment alternatives in the focus area. 
to appoint stops to the alignment alternatives. Where to position stops is dominated by the same factors as for the alignment except that it is the number of stops that affect the travel time. The stop positioning itself can be subject to generating alternatives since one alignment can have multiple stop patterns (different positioning but also different number of stops). However, this issue is not addressed in this paper.

\subsection{Case example: stop positioning on alternatives}

There are many options how to position stops on the three alignment alternatives. The positioning has been performed using travel potential maps (see figure 2), by securing transfers to other public transport lines and by common criteria of average stop distances (approximately 700 meters). All in all this gives a "best criteria" stop positioning on the three alignment alternative as seen in seen in figure 4.

\section{Evaluation of alignment alternatives}

In the end, only one final railway alignment can be implemented in the public transport system so when there are multiple alignment alternatives the best has to be chosen. To find the best alignment alternative, decision support that includes travel demand can be useful. It is also important to take possible transfers into account since they can supply a non-negligible part of the passengers especially in terminals. Unfortunately, transfers cannot easy be joint into a methodology with travel demand and the affect of transfers must, therefore, be assessed apart from the travel demand assessments.

In the travel demand methodology there are different computerised GIS based approaches depending on the desired level of detail and accuracy of the analysis. The approaches include buffer analysis and overlay analysis to apply travel demand data within defined buffers surrounding the railway where the buffer approach determines the level of detail of the analysis.

Regarding the buffer analysis a distance of 350 meters is used for all buffers in the case example. 350 meters has been chosen since studies (e.g. [3] and [4]) indicate a willingness to walk to LRT stations at about that distance. However, other distances could easily have been chosen and implemented too. The three different approaches are presented in the following.

\subsection{Corridor analysis}

A simple but straight-forward approach is to investigate corridors of urban railway alignments. This can be done by the line potential approach. The line potential approach simply investigates travel potential within a whole corridor of an alignment. Corridors of the three alternatives can be seen in figure 5 .

An overlay analysis gives the travel potential within each corridor and the results can be listed in a table (see table 1). 
Table 1: Corridor analysis - travel potential within the corridors of the three alignment alternatives - best alternative highlighted.

\begin{tabular}{|c|c|c|c|}
\hline & $\begin{array}{c}\text { Length } \\
{[\mathrm{km}]}\end{array}$ & Travel Potential & $\begin{array}{c}\text { Travel Potential / } \\
\text { Length }\end{array}$ \\
\hline Alt 1 & 8.00 & 62,808 & 7,851 \\
\hline Alt 2 & 6.38 & 76,964 & 12,063 \\
\hline Alt 3 & 6.39 & 61,269 & 9,588 \\
\hline
\end{tabular}

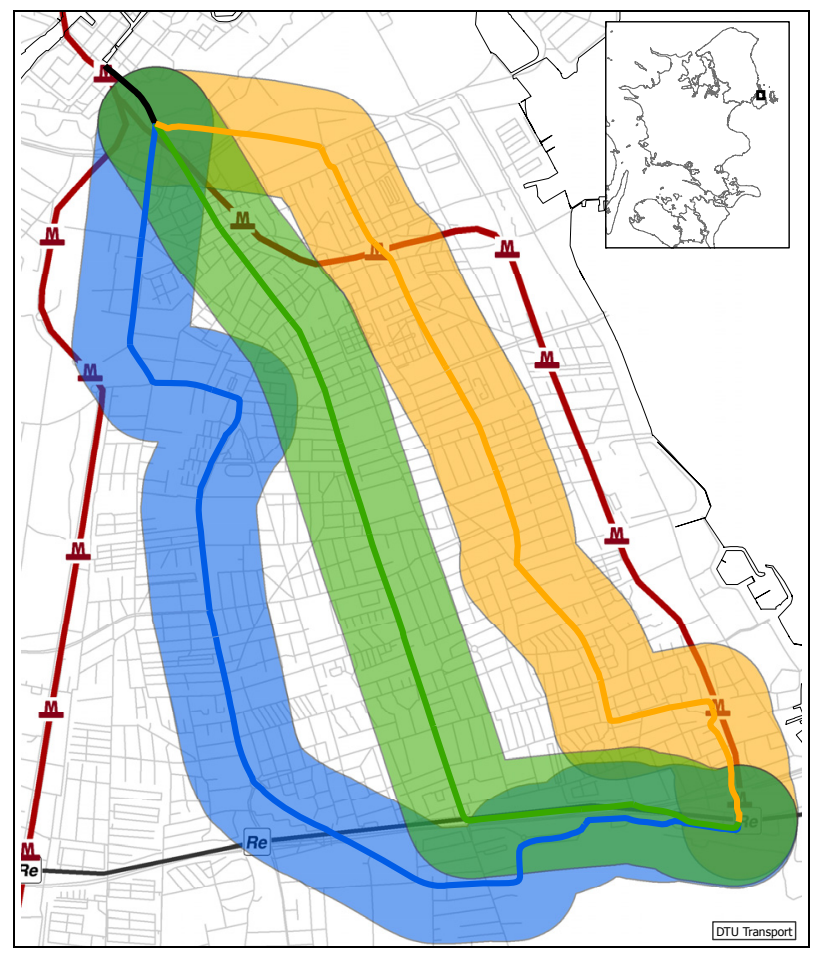

Figure 5: Corridors of the three alignment alternatives (within an Euclidean distance of 350 meters from alignments).

highest travel potential per length and is, therefore, regarded as the alternative best suited for selection.

Corridor Analysis is not a fully accurate approach since it is only possible to access a railway line at defined points (stops). However, corridor analysis still gives a good indication of travel demand for an alignment and it can be performed before positioning of stops and is, therefore, available as an easy-toimplement decision support tool in an early planning phase with many potential alternatives. 


\subsection{Catchment area analysis}

A more detailed approach is to include stops and investigate catchment areas of urban railway alignments through catchment area analysis. Catchment area analysis can determine travel potential within circular catchment areas of stops on an alignment. Catchment areas for the three alignment alternatives and their proposed stops can be seen in figure 6 .

An overlay analysis gives the travel potential within catchment areas of each alternative and the results can be listed in as in table 2 .

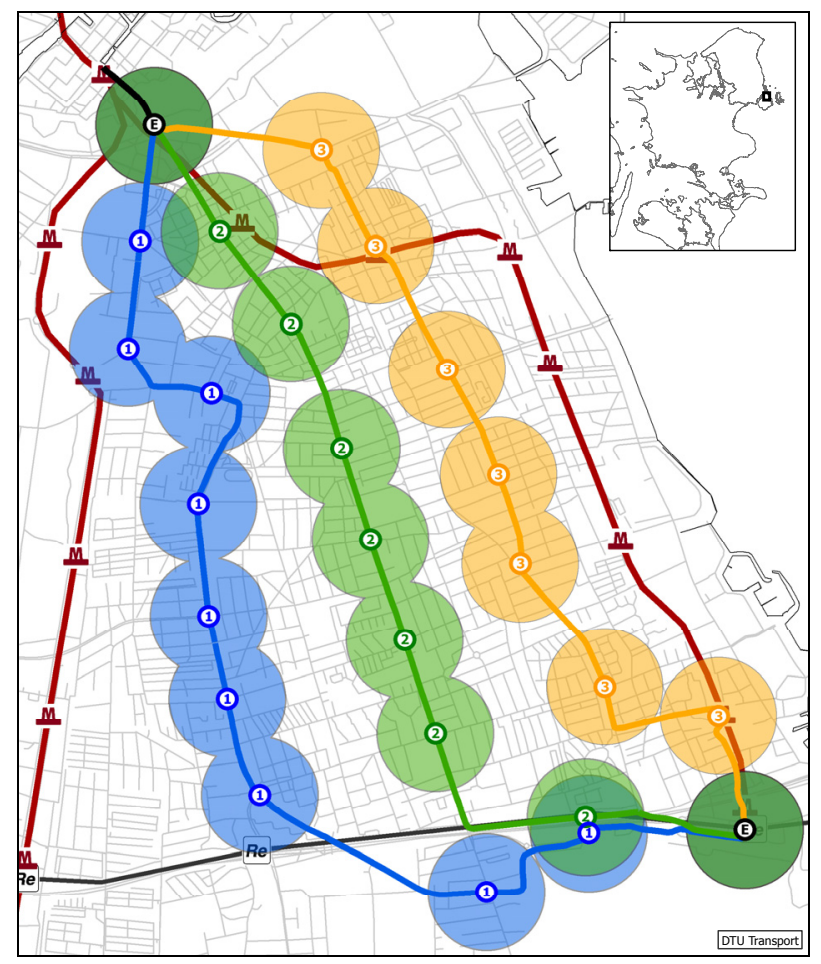

Figure 6: Catchment areas of stops on the three alignment alternatives (within a Euclidean distance of 350 meters from stops).

Table 2: $\quad$ Catchment area analysis - travel potential within circular catchment areas of stops on the three alignment alternatives - best alternative highlighted.

\begin{tabular}{|c|c|c|c|c|c|c|c|}
\hline & $\begin{array}{c}\text { Length } \\
{[\mathrm{km}]}\end{array}$ & Stops & $\begin{array}{c}\text { Avg.stop } \\
\text { distance } \\
{[\mathrm{km}]}\end{array}$ & $\begin{array}{c}\text { Travel } \\
\text { Potential }\end{array}$ & $\begin{array}{c}\text { Travel } \\
\text { Potential // } \\
\text { Length }\end{array}$ & $\begin{array}{c}\text { Travel } \\
\text { Potential / } \\
\text { Stop }\end{array}$ & $\begin{array}{c}\text { LinePot } \\
\text { utili } \\
\text { sation }\end{array}$ \\
\hline Alt 1 & 8.00 & 11 & 0.73 & 49,865 & 6,233 & 4,533 & $79 \%$ \\
\hline Alt 2 & 6.38 & 9 & 0.71 & 58,200 & 9,122 & 6,467 & $76 \%$ \\
\hline Alt 3 & 6.39 & 9 & 0.71 & 45,656 & 7,145 & 5,073 & $75 \%$ \\
\hline
\end{tabular}


Travel potential per length and travel potential per stop is most relevant for comparison between alternatives that are not equally long or have equal number of stops. As seen in table 2, Alternative 2 has the highest travel potential per length and per stop hence regarded as the best alternative. However, Alternative 1 has a higher line potential utilisation meaning it better utilises the travel potential of the corridor.

Since catchment area analysis is conducted on the actual access/egress points of public transport (the stops) it is a much more precise approach than corridor analysis thus providing a more accurate decision base. However, it also demands more work since the stop positioning has to be performed prior to the analysis. Catchment area analysis is, therefore, suitable for more thorough and realistic analysis of alignments in a later planning phase where the number of alternatives are low.

\subsection{Service area analysis}

A refinement of the catchment areas of stops is service areas. Service areas are based on searches in street and path networks and are, therefore, more realistic in terms of actual travel distances for the feeder traffic (for more information about

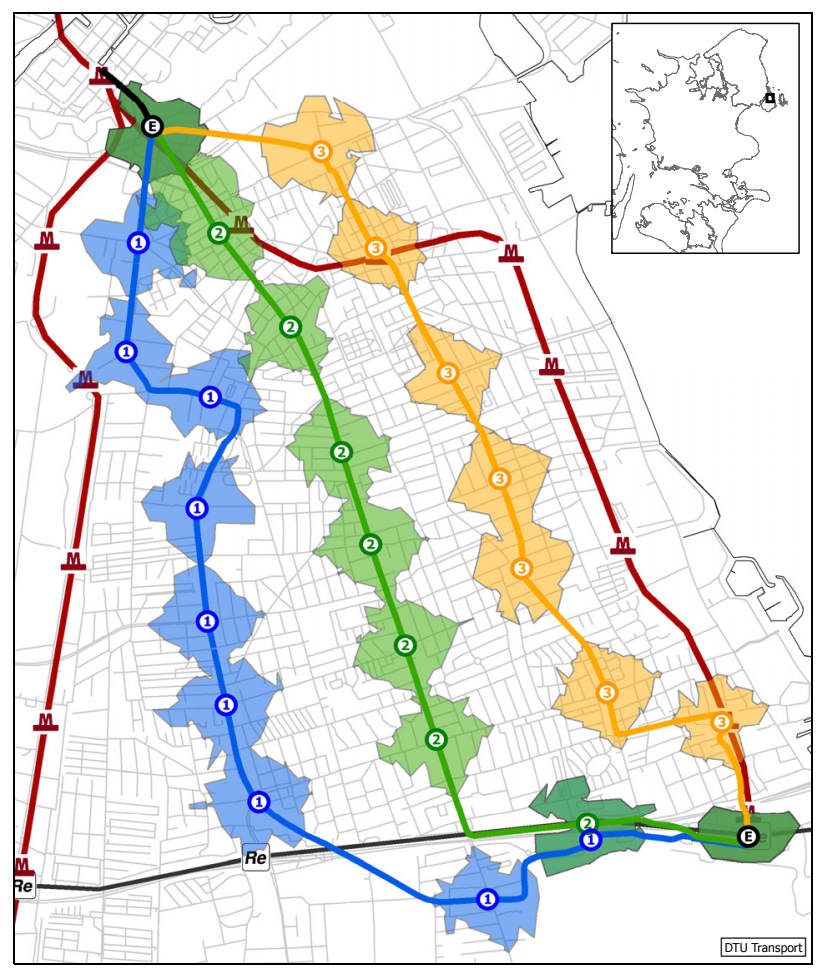

Figure 7: Service areas of stops on the three alignment alternatives (within a street network search distance of 350 meters from stops). 
service areas see [5] and [6]). Service areas of stops on the three alignment alternatives and their stops can be seen in figure 7 .

An overlay analysis gives the travel potential within service areas of each alternative and the results can be listed in as in table 3 .

Table 3: $\quad$ Service area analysis - travel potential within service areas of stops on the three alignment alternatives - best alternative highlighted.

\begin{tabular}{|c|c|c|c|c|c|c|c|c|}
\hline & $\begin{array}{c}\text { Length } \\
{[\mathrm{km}]}\end{array}$ & Stops & $\begin{array}{c}\text { Avg.stop } \\
\text { distance } \\
{[\mathrm{km}]}\end{array}$ & $\begin{array}{c}\text { Travel } \\
\text { Potential }\end{array}$ & $\begin{array}{c}\text { Travel } \\
\text { Potential } \\
\text { / Length }\end{array}$ & $\begin{array}{c}\text { Travel } \\
\text { Potential } \\
\text { / Stop }\end{array}$ & $\begin{array}{c}\text { LinePot } \\
\text { Utili } \\
\text { sation }\end{array}$ & $\begin{array}{c}\text { CA } \\
\text { Utili } \\
\text { sation }\end{array}$ \\
\hline Alt 1 & 8.00 & 11 & 0.73 & 33,568 & 4,196 & 3,052 & $53 \%$ & $67 \%$ \\
\hline Alt 2 & 6.38 & 9 & 0.71 & 41,478 & 6,501 & 4,609 & $54 \%$ & $71 \%$ \\
\hline Alt 3 & 6.39 & 9 & 0.71 & 27,377 & 4,284 & 3,042 & $45 \%$ & $60 \%$ \\
\hline
\end{tabular}

As seen in table 3 Alternative 2 has the highest travel potential per length and per stop making it the best suitable alternative. It also utilises the travel potential of the corridor and catchment areas (CA) best.

Since the service area analysis is based on the actual travel distances of the feeder traffic it is the most detailed and accurate approach. It also requires more detailed input data, especially the street and path network. For best performance of the approach (and to make it as realistic as possible) all areas surrounding stops must be scrutinized for data availability and realism. The service area approach can be used to conduct more detailed analysis of alignment alternatives than the catchment area approach but it also requires more preparation of data and is, therefore, best suited for analysis with high accuracy requirements usually conducted in a late planning phase. The largest applied benefit of the approach is its ability to describe the effect of changes in the street and path network surrounding stops; thereby being suitable for analyses of accessibility to public transport (see [5]).

\subsection{Results and discussion}

From the case example it is seen how Alternative 2 turned out to be the best using all three approaches. This is a strong indication that this alternative is the best one when investigating travel potential. It also shows a consistency between the approaches. However, there may be cases where there will be differences between the results of the approaches and where e.g. the stop positioning of a poor alternative can utilise the travel potential of the corridor so good that it will show better results once analysis of stops are taken into account. This never becomes an issue in the case example since Alternative 2 simply is much better than the other alternatives.

The evaluation criteria are mostly based on the travel potential per length and travel potential per stop. A key performance indicator taking both length and number of stops into account can also be desirable. Such an indicator could be based on e.g. construction cost or a travel time based operating cost both implementing a length and a stop depending contribution. A key performance 
indicator like that can provide a more clear and understandable overview of the alternatives and ensure that a long twisted alignment with many stops will not be much better than a shorter straight alignment. This can be illustrated by comparing the next best alternatives in the case example. Alternative 1 is longer, has more stops, and as a result this alternative has higher travel potential compared to alternative 3 . However, taking both travel potential per length and travel potential per stop into account the alternatives seem quite equal. Using a common key performance indicator may even prove that Alternative 3 is better than Alternative 1 depending on the weights applied to length and to stops.

The case example shows how the approaches can be used as decision support tools when examining different alignments with the same terminal stations. However, the methodology may also be used to examine alignments with different terminal stations but the more different the alignment alternatives are, the more careful one must be to achieve comparable results. The methodology cannot compare different types of service and its effect on passenger attractiveness, e.g. fast service with few stops (end to end service or shuttle service) and slower service with more stops (local service). The methodology should, therefore, only be used to compare alternatives of roughly the same type of service. Travel time for each alternative and comparison with the existing service can be included in the evaluation criteria as well, but whether the service of an alternative is the best suitable for passengers must be evaluated through the more time consuming traffic modelling. Otherwise the desired service characteristics of the railway must be decided on before making the alternatives.

\section{Conclusions}

A quick-to-implement decision support methodology based on travel demand can be used to select the best alignment of an urban railway between multiple alignment alternatives. The methodology is based on computerised GIS analysis and comes in different approaches depending on the level of detail of the investigations. In an early screening phase the simple corridor analysis can be used to deselect the less suited alignment alternatives. In a more advanced planning phase where stops are appointed to the alignment alternatives the catchment area analysis can be used as a realistic foundation for selecting the best alignment. An even more detailed approach is to refine the catchment area analysis using service areas of stops as base for the selection. The detailed level of the service area approach also makes it relevant for analysis of the accessibility to each stop but it also demand more detailed input data.

The output of all approaches is travel potential within the defined buffers surrounding the railway line and it provides an overall sound decision support in the alignment selection process. However, a factor such as transfer to other public transport lines must not be ignored since large passenger volumes can be generated from transfers especially in terminals. But as the main part of the decision support of alignment alternatives the methodology is essential and can relatively easy be applied in the planning process of urban railways. 


\section{References}

[1] Andersen, J.L.E., Landex, A. \& Nielsen, O.A., Light rail project in Copenhagen - the Ring $2 \frac{1}{2}$ corridor, Proc. of the Annual Transport Conference at Aalborg University, 2006.

[2] Jacobsen, B. \& Larsen, F., Catchment area and transport modelling (Stationsoplands- og trafikmodelberegninger), Proc. of the Annual Transport Conference at Aalborg University, 1999 (in Danish).

[3] O'Sullivan, S. \& Morral, J., Walking Distances to and from Light-Rail Transit Stations, Transport Research record, 1538, pp. 19-26, 1996.

[4] Christiansen, H., Laursen, J.G. \& Jørgensen, H.E., Feeder geography at bus stops (Tilbringergeografi ved busstoppesteder), Institute of Planning, DTU, 2000 (in Danish).

[5] Landex, A., Hansen, S. \& Andersen, J.L.E., Examination of catchment areas for public transport, Proc. of the Annual Transport Conference at Aalborg University, 2006.

[6] Andersen, J.L.E. \& Landex, A., Catchment areas for public transport, Proc. of the Urban Transport Conference at Malta, 2008. 